\title{
MODELOS ECONÓMICOS: ¿REPRESENTACIONES AISLADAS O CONSTRUCCIONES FICTICIAS?
}

\author{
ECONOMIC MODELS: ISOLATED \\ REPRESENTATIONS OR FICTIONAL \\ CONSTRUCTIONS?
}

\author{
LEONARDO IVAROLA* \\ Universidad de Buenos Aires
}

Resumen: En la moderna filosofía de la economía existen dos posturas principales respecto de la naturaleza y funcionamiento de los modelos económicos: los modelos como representaciones aisladas y como construcciones ficticias pero creíbles. Ambas posturas serán examinadas en el presente trabajo, y contrastadas con aquello que se denominará "procesos socioeconómicos basados en expectativas". Se verá que estos últimos no responden a una lógica mecanicista o de factores estables, sino más bien a una lógica de árboles de posibilidades. Por tal razón, se argumentará que los modelos económicos, al menos en su mayoría, no deberían ser interpretados como representaciones de mecanismos y/o capacidades aisladas, sino como estructuras que, de existir en el mundo real, serán generadoras de tendencias. A pesar de que la temática abordada se limita al estudio de los modelos económicos, sus implicancias pueden extenderse a diferentes ramas de las ciencias sociales.

Palabras Clave: modelos económicos, aislamiento teorético, construcción ficticia, procesos socioeconómicos

* Leonardo Ivarola. Centro de Investigaciones en Epistemología de las Ciencias Económicas, Facultad de Ciencias Económicas, Universidad de Buenos Aires. E-mail: ivarola@economicas.uba. ar. Argentina. 
АвSTRACT:In modern philosophy of economics there are two main positions in relation to the nature and functioning of economic models: models as isolated representations and models as fictional but credible constructions. Both positions are examined in this paper and contrasted with what I will call "expectation-based socioeconomic processes." I will show that these processes are not compatible with a mechanistic logic or a logic of stable causal factors, but that they are compatible with the logic of possibility trees. Because of this, I will argue that - at least in most cases - economic models should not be interpreted as representations of isolated mechanisms or capacities, but as structures which, if they existed in the real world, would be able to generate tendencies. Although the present topic is limited to the analysis of economic models, its implications may be extended to different branches of the social sciences.

KEYwORDs: economic models, theoretical isolation, fictional construction, socioeconomic processes

\section{Introducción}

Dentro de la filosofía de las ciencias sociales en general y de la economía en particular existe consenso en concebir al mundo real como un sistema complejo (García, 2006; Hayek, 1981; Mill, 1843; Mäki, 1992) o desordenado (Cartwright, 1999a). Existe una miríada de causas que operan permanentemente en este mundo, genera tanto efectos potenciadores como contrarrestantes de los factores causales que pretenden descubrirse. Para hacer frente a esta problemática, la ciencia está obligada a emplear métodos de modificación o de deformación del mundo, a los efectos de hacer a éste más inteligible. Estos intentos de brindar mayor inteligibilidad involucran construcciones distorsivas, como es el caso de los modelos económicos.

Hoy en día, la construcción de modelos teoréticos se ha expandido tanto en la economía, que se ha llegado a decir que "hacer economía es hacer modelos" (Mäki, 2002b, p. 10). La utilidad de esta investigación teorética reside en que se puede aprender "algo" del mundo real a través de los modelos. En general, la filosofía estándar de la economía apoya la idea de que los modelos proveen una plantilla lógica y abstracta que ayuda a organizar los pensamientos del analista. El modelo ayuda al economista a aislar factores causales teoréticamente y a resolver largas cadenas causales influenciadas por los numerosos elementos que interactúan en una economía. A través del uso de un modelo, el economista puede 
experimentar, al menos lógicamente, la producción de diferentes escenarios, así como también intenta evaluar el efecto de las opciones de políticas alternativas (Morgan, 2012. Véase también Morgan y Morrison, 1999; Mäki, 2002b, 2005).

Tradicionalmente se ha concebido a los modelos como representaciones del mundo, y el problema subyacente a este modo de interpretación consistía en determinar si resultaban o no adecuadas (esto es, falsas, verdaderas o aproximadamente verdaderas respecto de la realidad representada). Sin embargo, no existe consenso respecto de cómo se ejerce esta función representadora. Por un lado, el enfoque semántico concibe a la representación como una relación diádica entre entidades lingüísticas y el mundo (Hausman, 1992). En contraste, el enfoque pragmático destaca el rol del científico y sus respectivos propósitos en la actividad representativa (Giere, 2004; Mäki, 2009).

Por lo general, dentro de la concepción "representacional” prevalece la idea de que los modelos económicos funcionan como aislamientos teoréticos de factores tales como los mecanismos y las capacidades (Mäki, 1992, 2005, 2009; Cartwright, 1989, 1998). Este método de aislamiento consiste en "separar" un factor causal de cualquier elemento perturbador, de modo tal que dicho factor pueda encontrarse en "estado puro". Sin embargo, esta "separación" no se hace empíricamente (como sí sucede con métodos tales como la prueba controlada aleatoria), sino teoréticamente o dentro del modelo. En particular, el modelo funciona como un "sistema sustituto" en el cual podemos obtener conocimiento si tenemos éxito en aislar correctamente un mecanismo, capacidad o tendencia.

Actualmente existe dentro de la filosofía de la economía una rica discusión respecto del rol de los modelos en la actividad investigativa, y se han propuesto enfoques alternativos al representacional. Por ejemplo, uno de los enfoques en boga en la literatura moderna es el de entender a los modelos como "ficciones". Un modelo como entidad ficticia no busca representar la realidad ni aproximarse a la misma, sino comprenderla a través de un relato sencillo que capture algunas de sus características esenciales. Dentro de esta categoría se han propuesto diferentes alternativas: modelos como caricaturas (Gibbard y Varian, 1978), como economías análogas (Boumans, 1997; 2006; Lucas, 1980; Cartwright, 1999b), y como mundos creíbles (Sugden, 2002, 2009). ${ }^{1}$

${ }^{1}$ En realidad, la literatura sobre modelos económicos es abundante, y puede llevar a una tipología mucho más amplia que las clases de modelos examinadas en el presente trabajo. En general, a los modelos económicos se los han conceptualizado de diferentes maneras: como media- 
En el presente trabajo se examinarán ambas corrientes a la luz de lo que denominaremos "procesos socioeconómicos basados en expectativas" o PSBE. Se mostrará que, dada su naturaleza óntica de responder a la lógica de los árboles de posibilidades, los modelos económicos - al menos en su gran mayoría - no pretenden aislar mecanismos o capacidades, sino construir un sub-sistema en donde la estructura prevaleciente permita la inferencia de resultados. Tanto la estructura asumida como la inferencia alcanzada pueden brindar información significativa respecto de la realidad fenoménica, ya que se estarían haciendo aseveraciones respecto de las condiciones a cumplir en el mundo real para que en este mismo puedan emerger los resultados inferidos en el modelo.

\section{Modelos como aislamientos teoréticos}

El método de "aislar" factores causales (tales como las capacidades y los mecanismos) surge como consecuencia de la complejidad de los sistemas en los que se pretende descubrir dichos factores. Este método consiste en separar un factor causal de cualquier elemento perturbador, de modo tal que dicho factor pueda encontrarse en "estado puro". En este proceso de separación, el universo relevante queda dividido en dos subconjuntos o campos separados (Hands, 2001).

"In an isolation, something, a set X of entities, is "sealed off" from the involvement or influence of everything else, a set $\mathrm{Y}$ of entities; together $\mathrm{X}$ and $\mathrm{Y}$ comprise the universe. The isolation of $\mathrm{X}$ from $\mathrm{Y}$ typically involves a representation of the interrelationships among the elements of $X$. Let us call $\mathrm{X}$ the isolated field and $\mathrm{Y}$ the excluded field. It should be obvious that any representation involves isolation: isolation is ubiquitous in human cognition.” (Mäki, 1992, p. 321. Énfasis en original).

\footnotetext{
dores (Haavelmo 1944; Morgan and Morrison, 1999), como fórmulas abiertas para proponer hipótesis (Alexandrova 2008; Guala 2005), como medios para contar historias (Dow 2002; Morgan, 2002), y como analogías (Hesse 1966; McMullin, 1968). Marqués (2014) ha propuesto una tipología de modelos económicos, diferenciado entre modelos normativos (que pueden o no ser prescriptivos), modelos teóricos (o exploratorios) y modelos fácticos (que pueden ser de estado actual, de estado final, procedurales o tendenciales). Morgan y Knuuttila (2012) han identificado dos rutas principales en relación con el status epistémico de los modelos económicos: como idealizaciones y como construcciones. En este último caso, algunos han sido comprendidos como representaciones, otros como entidades ficticias o artificiales, y otros como entidades autónomas que median entre la teoría y los datos. En el presente trabajo se utilizará la categorización propuesta por Morgan y Knuuttila (2012) - modelos como idealizaciones y como construcciones - y, por razones de simplicidad o de espacio, sólo se examinarán las construcciones ficticias.
} 
En la visión de Mäki, la abstracción es un tipo particular de aislamiento. "En una abstracción, un universal o cuasi-universal es aislado de ejemplificaciones particulares" (1992, p. 322). Tomemos como ejemplo la función de demanda $Q^{d}=f(P)$, donde $Q^{d}$ es la cantidad demandada y $P$ el precio. Dicha función está basada en una abstracción, ya que denota un universal (o cuasi-universal) que no hace referencia a ninguna instancia específica. Supongamos ahora que la función de demanda toma una forma más específica, por ejemplo, $Q^{d}=a+b$.P. Aunque también esté basada en una abstracción, esta segunda función será menos abstracta que la primera. Más aun, si determinásemos los valores de $a$ y de $b$, esta tercera ecuación sería todavía menos abstracta que la anterior. Esto permite mostrar que existen niveles de abstracción, los cuales se encuentran en relación inversa con el proceso de concretización (también llamado "de-aislamiento"): cuanto mayor sea el grado de abstracción, menor será el nivel de concretización, y cuanto menor sea el grado de abstracción, mayor será el nivel de concretización. A esta clase de aislamientos Mäki la denomina "aislamiento vertical", y se contrapone al "aislamiento horizontal". En este último caso existe un proceso de separación, pero el grado de abstracción permanece inalterado. Por ejemplo, dada una ecuación $Y=f\left(x_{1}, \ldots, x_{n}\right)$, la aislación puede consistir en separar el efecto que $x_{1}$ causa sobre $Y$ del resto de las $x_{i}$, esto es, $Y=f\left(x_{1}\right)$.

El modo en que se genera el proceso de aislamiento puede hacerse de dos maneras diferentes, dependiendo de si el sistema involucrado es concreto o conceptual. El aislamiento material es un proceso por el cual un factor causal presente en un sistema concreto es separado de un conjunto de posibles factores perturbadores. Dentro de esta clase se pueden diferenciar dos categorías: el aislamiento experimental y el aislamiento espontáneo. El primero "es manifiesto cuando un sistema real, relación, proceso o característica, basado en una intervención causal en el proceso que ocurre en el mundo, es materialmente cerrado de la participación o interferencia causal de otras entidades reales" (Mäki, 1992, p. 325). El aislamiento espontáneo es, por el contrario, un proceso que ocurre por la acción misma de la naturaleza, esto es, sin intervención alguna del hombre.

Los aislamientos materiales son muy comunes en las ciencias naturales. Sin embargo, puesto que la noción de "sistema complejo" se aplica tanto al reino de lo social como de la naturaleza, los aislamientos experimentales son los más frecuentes. Tomemos como ejemplo el descubrimiento del bacilo de Koch como agente causal de la tuberculosis. La tuberculosis (también llamada tisis) era una enfermedad muy difundida en los países de Europa, y producía miles de víctimas todos los años, independientemente de su condición física o social. Las causas 
de esta enfermedad se desconocían, y esto llevó a los investigadores a postular diferentes hipótesis. Sobre la base de múltiples observaciones en su laboratorio, Koch descubrió que existía una bacteria - a la cual denominó "bacilos tuberculosos" - que estaba presente en todos los individuos que padecían esa enfermedad. Para probar esto, Koch aisló el agente patógeno en un medio de cultivo puro. Posteriormente se les inyectó el cultivo de los bacilos a animales en diferentes áreas del cuerpo (abdomen, bajo la piel, en la sangre, etc.). Sin excepción, cada uno de ellos se volvió tuberculoso.

Ahora bien, existen algunas ocasiones en las cuales las posibilidades de llevar a cabo experimentos de laboratorio son muy remotas. Por tal razón, los científicos apelan al aislamiento causal a través de los experimentos mentales, también denominados aislamientos teoréticos. En un aislamiento mental o teorético se intenta separar un factor causal, pero no en el mundo real (o sistema concreto), sino dentro de un modelo. Siguiendo a Mäki, el aislamiento mental "es manifiesto cuando un sistema real, relación, proceso o característica, basado en una operación intelectual en la construcción de un concepto, modelo o teoría, está cerrado de la participación o impacto de otras características de la situación" (1992, p. 325).

En términos generales, la aislación teorética es una operación intelectual de características semejantes a la experimentación científica: ambas involucran un proceso de intervención a través de la clausura de posibles factores perturbadores. Por esta razón, Mäki concibe a las aislaciones teoréticas como experimentos mentales. Los modelos son construidos para crear un mini-mundo en contraste al complejo y desordenado maxi-mundo. Los aislamientos se logran a través de la manipulación de ciertas entidades. Lo que diferencia un experimento mental de uno material depende de qué elemento ha de ser manipulado:

"While material experimentation employs causally effected controls, theoretical modelling uses assumptions to effect the required controls. Assumptions are used to neutralise, in the model worlds, the involvement of other things by assuming them to be constant, absent, of zero strength, negligibly small, in a normal state, within certain intervals, and so on (...) The structure of experimentation, involving controls and isolation, is the same, while what is different is the way these controls and isolations are effected: by way of thinking and assuming, and by way of material or causal manipulation.” (Mäki 2005, pp. 308-309). 
Existen diferentes modos de lograr una aislación teorética. Mäki (1992) propone dos técnicas para alcanzarla: la omisión y la idealización. La idealización es un tipo particular de aislamiento horizontal, en donde se establece el valor límite de una variable en 0 (cero) o $\infty$ (infinito). Idealizaciones de esta clase abundan en economía. Ejemplos de esta categoría son la infinita divisibilidad de bienes, pleno empleo, elasticidad perfecta (o infinita), costos de transacción nulos, etc. Otro ejemplo son los modelos macroeconómicos de economías cerradas. En estos últimos casos, tanto el monto de las importaciones como de las exportaciones son nulos. Este supuesto se introduce con el propósito de simplificar el análisis, esto es, para investigar la contribución de alguna variable macroeconómica fuera de la influencia de factores causales propios del comercio internacional.

La omisión, en cambio, es "un tipo de idealización implícita en la que el factor omitido no es mencionado para nada en la presentación de la teoría o modelo" (Mäki, 1992, p. 330). En la omisión no se considera que el valor de una variable sea nulo, sino que ni siquiera es relevante para el análisis en cuestión. Comparemos el caso de la economía neoclásica y la institucionalista. La economía neoclásica omite la influencia causal de una miríada de cosas, desde reglas legales y rutinas organizacionales a la velocidad de Júpiter y el número de cromosomas característicos de las orquídeas, mientras que la economía institucionalista omitiría sólo de esos factores la influencia de Júpiter y de las orquídeas (Mäki, 1992).

\section{Modelos como ficciones}

La primera pregunta que uno debería hacerse al examinar los modelos económicos es: ¿los economistas efectivamente pretenden aislar factores causales relevantes del mundo real? ¿ $\mathrm{O}$, por el contrario, sus modelos son construcciones ficticias que, aunque sirven para hacer diferentes clases de inferencias sobre eventos del mundo real, no deben ser interpretados como representaciones de esa misma realidad?

Dentro de la filosofía de la economía, uno de los primeros trabajos en concebir a los modelos como sistemas artificiales o entidades ficticias es el de Gibbard y Varian (1978), quienes aseveran que muchos de los modelos teoréticos deberían ser entendidos no como aislamientos ni aproximaciones, sino como caricaturas. "Frecuentemente los supuestos de un modelo no son elegidos para aproximarse a la realidad, sino para exagerar o aislar alguna característica de ésta” (p. 673). El propósito del modelo no es entonces acercarse a la realidad, sino comprenderla 
a través de un relato sencillo que capture algunas de sus características sobre la base de su exageración o distorsión.

Por caricatura Gibbard y Varian quieren decir "no sólo que la aproximación hecha es tosca y simple, sino que el grado de aproximación no es una consideración importante en el diseño del modelo" (p. 673). La investigación científica comienza por lo general distinguiendo algún aspecto del mundo real. Luego el teórico construye un modelo que pueda explicar el fenómeno observado. Sin embargo, esta realidad a la que se enfrenta el teórico es compleja, por lo que la construcción del modelo no estará dirigida a obtener la aproximación más cercana a la realidad, sino a contar un relato simple que capture algunas de sus características más prominentes. Gibbard y Varian utilizan como ejemplo el modelo de transferencia inter-generacional de Samuelson. El propósito de dicho modelo radica en evaluar las virtudes y defectos de programas que involucran esta clase de transferencias, como pueden ser los programas de seguridad social. El modelo supone una población uniforme - que puede ser reducida a un agente representativo -, los agentes viven solo dos periodos (en uno trabajan y en otro se retiran), etc. Estos supuestos están claramente alejados de la realidad. Sin embargo, deben entenderse simplemente como ficciones convenientes para la comprensión e inferencia de resultados, no como aproximaciones de la realidad.

La utilidad de un modelo como caricatura para la comprensión de una situación está relacionada con la robustez de las conclusiones. Un resultado es robusto cuando éste no depende de los detalles de los supuestos. En general, se puede decir que los supuestos del modelo intervienen en la caricaturización de una situación. Al cambiar los supuestos, la caricatura va a cambiar. Por consiguiente, un resultado o conclusión va a ser robusto cuando no se vea alterado ante cambios en las caricaturas. Esto justifica aún más la idea de que lo relevante en la caricaturización no es la descripción de la caricatura en sí misma, sino el resaltar algún aspecto particular de la realidad.

Quizás el enfoque más controversial acerca de los modelos como ficciones es el de "mundos paralelos creíbles" de Robert Sugden (2002, 2009). De acuerdo con el autor, los modelos describen mundos contrafácticos que paralelan con el mundo real. Los modelos son, en otras palabras, mundos creíbles.

En el mundo modelo los resultados se obtienen de manera deductiva sobre la base de un conjunto de premisas. Si esto fuera todo el aporte, los modelos no proporcionarían información acerca del mundo real; simplemente afirmarían qué 
sucederá bajo condiciones muy específicas (véase Cartwright, 2009). Sin embargo, Sugden considera que los hacedores de modelos obtienen algo mucho más general que meros teoremas que sólo serían verdaderos en mundos contrafácticos. Sus resultados son generalizaciones que, siendo verdaderas en el modelo (ya que son el resultado de inferencias deductivas), podrían ser también "verdaderas" en el mundo real. Empero, estas hipótesis acerca de eventos que pueden acontecer en el mundo real no son inferidas de los modelos a partir de razonamientos deductivos.

¿Cómo puede ser hecha esta transición? Sea $\mathrm{R}$ una regularidad que puede o no ocurrir en el mundo real, y sea $\mathrm{F}$ un conjunto de factores causales que pueden o no operar en el mundo real. De acuerdo con Sugden (2002), los economistas parecen razonar de la siguiente manera:

\section{Esquema 1: explicación}

- En el mundo modelo, R es causado por F

- F opera en el mundo real

- R ocurre en el mundo real

Ergo, hay razones para creer que

- En el mundo real, $\mathrm{R}$ es causado por $\mathrm{F}$

\section{Esquema 2: Predicción}

- En el mundo modelo, R es causado por F

- F opera en el mundo real

Ergo, hay razones para creer que

- R ocurrirá en el mundo real

\section{Esquema 3: Abducción}

- En el mundo modelo, R es causado por F

- R ocurre en el mundo real

Ergo, hay razones para creer que

- F opera en el mundo real

En cada uno de los esquemas mencionados, el "ergo" requiere un salto inductivo. Para Sugden, el razonamiento inductivo es aquel que conduce de proposiciones específicas a otras más generales. Aquí, la proposición específica es justamente la aserción de que R es causada por F en el mundo modelo. Ahora bien, 
el salto inductivo no radica en ir de un caso particular en el mundo real a una aserción general en éste. En contraste, el salto consiste en ir del caso particular explicitado en el mundo modelo - el cual es considerado un mundo posible o imaginario - al caso general en el mundo real.

La siguiente pregunta que se hace Sugden es cómo poder justificar estas inferencias inductivas, la cual va a responder a través de su concepción de mundos creíbles. El razonamiento inductivo trabaja encontrando alguna regularidad $\mathrm{R}$ en una colección de observaciones $\mathrm{x}_{1}, \ldots, \mathrm{x}_{\mathrm{n}}$, y luego se infiere que $\mathrm{R}$ también se encontrará en un conjunto general de fenómenos $\mathrm{S}$, el cual no solo contiene a la colección $\mathrm{x}_{1}, \ldots, \mathrm{x}_{\mathrm{n}}$, sino también a una serie de elementos que no han sido todavía observados (Sugden, 2002). ${ }^{2}$

Ahora bien, este tipo de razonamientos cobrará relevancia en la medida en que los elementos de $\mathrm{S}$ sean similares. Dicha similaridad va a ser entendida en términos de analogía entre factores causales: lo que los dos mundos - el "real" y el "modelo" - tienen en común es que esos factores están presentes en ambos. En este marco, el mundo real puede ser entendido como un modelo mucho más complejo que los modelos teoréticos: "es el caso límite del proceso de reemplazar los supuestos simplificadores del modelo original con especificaciones realistas crecientes" (Sugden 2002, p.129). Si es posible hacer inferencias inductivas desde un modelo simple y sencillo a variantes cada vez más complejas, entonces este proceso puede continuar hacia el caso más extremo de complejidad: el mundo real como modelo.

Es menester entonces diferenciar entre modelos naturales - que se refieren a estados del mundo real - y modelos teoréticos o conceptuales. En numerosas ocasiones la inferencia inductiva se realiza entre modelos naturales: si a cuatro perros que se les ha extirpado el páncreas desarrollaron diabetes, entonces a los próximos perros que se les extirpe el páncreas también van a desarrollar diabetes. Si los científicos hacen inferencias inductivas entre modelos naturales, entonces también debería ser viable hacer inferencias inductivas entre modelos teoréticos. Y puesto que ambos son modelos, luego también va a ser viable hacer estas mismas inferencias del mundo modelo al mundo real.

2 En otras palabras, si $\mathrm{R}$ está presente en $\mathrm{x} 1, \ldots, \mathrm{xn}$, entonces (inductivamente) $\mathrm{R}$ va a estar presente en cada uno de los elementos de S. 
La confianza en estas inferencias inductivas se gana siendo capaz de ver a los modelos relevantes como instancias de una cierta categoría, algunas de las cuales existen en el mundo real. Reconocemos el significado de la similaridad entre los mercados-modelos y los mercados reales aceptando que el mundo modelo podría ser real, en el sentido de describir un estado de cosas que sea creíble dado el conocimiento disponible (Sugden, 2002). ${ }^{3}$

El mundo modelo es evaluado entonces por su credibilidad. Esta última no es lo mismo que la verdad, sino que se asemeja a la verosimilitud o veracidad. Percibimos un mundo modelo como creíble cuando somos capaces de considerarlo como un mundo que podría ser real, no en el sentido de asignarle probabilidades subjetivas positivas al caso que es real, sino en el sentido de que sea compatible con lo que sabemos acerca de las causas que rigen los acontecimientos en el mundo real (Sugden, 2009). Un buen ejemplo de ello son las novelas. Una novela puede ilustrar un mundo posible o no, y eso dependerá de las características que comparta o se diferencie con la realidad. Una novela que describa agentes sobrenaturales o presente hechos anacrónicos hará que esa novela no sea tildada de "creíble" o "realista".

\section{Los Procesos Socioeconómicos Basados en Expectativas (PSBE)}

El enfoque de los modelos como representaciones aisladoras y el de construcciones ficticias difieren no sólo en sus aspectos epistémicos, sino también en los aspectos ontológicos. Mäki (así como también Cartwright, 1989, 1998) defiende la idea de un mundo gobernado por factores causales estables, a los cuales llama "mecanismos". Sugden no se compromete con esta postura. Este último simplemente entiende a los modelos como ficciones que pueden ser de utilidad a la investigación científica, en tanto dichos modelos sean creíbles, esto es, que el escenario descrito en el modelo sea congruente con nuestro conocimiento disponible. Sin embargo, no hay un compromiso con la idea de factor

3 Tal como señalan Xavier de Donato Rodríguez y Jesús Zamora Bonilla (2009), la idea de que el mundo creíble "podría ser real" no debe ser considerada tan literalmente. Los modelos frecuentemente contienen idealizaciones que, si se interpretaran literalmente, no son verdaderos (como por ejemplo los supuestos de continuidad, bienes homogéneos, etc.). Lo relevante del caso radica en que dichos supuestos no afecten materialmente los resultados inferidos, al mismo tiempo que vuelvan el análisis mucho más sencillo (Sugden, 2009). 
causal estable, capacidad o mecanismo. Estos pueden estar presentes en algunos modelos, pero su presencia o no-presencia no determina qué modelos van a ser más útiles para comprender el mundo, predecir fenómenos no ocurridos o implementar políticas.

Pensar en términos de mecanismos - y de factores estables/invariantes en general - es sugestivo, ya que proporciona una base teorética robusta para el cumplimiento de diferentes propósitos científicos. Sin embargo, puede ser cuestionable la idea de estabilidad o invarianza en ciencias sociales (y en la economía en particular). Las relaciones causales que estas ciencias estudian están mediadas por la acción humana, por lo cual este modo de pensamiento debe presuponer una conducta invariante por parte de los agentes y de las estructuras en las que éstos toman decisiones.

Mäki no examina la naturaleza de los mecanismos, y simplemente los entiende como factores causales o relaciones de dependencia (Mäki, 2009). Sin embargo, hay una rica discusión en la filosofía de la ciencia moderna respecto de los aspectos ontológicos y epistémicos de los mecanismos (véase, por ejemplo, Bunge, 2004, 2005; Glennan, 2002, 2008; Hedström y Swedberg, 1998a, 1998b; Hedström e Ylikoski, 2010), discusión que ha dado lugar a la denominada Nueva Filosofía Mecanicista (Skipper y Milstein, 2005). Dentro de esta escuela se sostiene como idea general que los mecanismos son factores estables que se manejan de manera automática una vez puestos en funcionamiento. La estabilidad significa que buena parte de las veces que se active $\mathrm{X}$ se obtendrá el resultado Y. La automaticidad significa que una vez activado $\mathrm{X}$, la secuencia se manejará sin necesidad de posteriores intervenciones para arribar a Y.

Sin embargo, otras corrientes alternativas consideran que, al pensar en términos de mecanismos socioeconómicos, se omite el carácter subjetivo de la acción humana:

"No "automatic" and "anonymous" forces actuate the "mechanism" of the market. The only factors directing the market and determining prices are purposive acts of men. There is no automatism; there are men consciously aiming at ends chosen and deliberately resorting to definite means for the attainment of these ends. There are no mysterious mechanical forces; there is only the will of every individual to satisfy his demand for various goods. There is no anonymity; there are you and I and Bill and Joe and all the rest. And each of us is engaged both in production and consumption. Each 
contributes his share to the determination of prices" (von Mises, 1949, pp. 311-312. Énfasis en original).

Una postura similar a la de von Mises será defendida en el presente trabajo. Los procesos que ocurren en los sistemas socioeconómicos no son producto de mecanismos que operan a nivel transfáctico (como sí sostiene, por ejemplo, Lawson (1997)). Los procesos dependen intrínsecamente de la acción humana, la cual es "vulnerable" a la información que recibe del mundo y al contexto que la rodea. En este sentido, el propósito de la presente sección apunta a abrir la "caja negra" de los procesos socioeconómicos y, en particular, de aquellos que involucran formación de expectativas, al cual denominaremos "procesos socioeconómicos basados en expectativas" (PSBE).

Básicamente, un PSBE es una secuencia de eventos conformada por tres clases de entidades. En primer lugar, una entidad emisora, que consiste en una señal que transmite información. En segundo lugar, una entidad receptora, que recibe e interpreta la información que la señal envía. Esta terminología está dominada por la naturaleza específica de la entidad mencionada en segundo término (los individuos), que en la presente interpretación de los PSBE adquiere un papel central. Finalmente, la actividad es la reacción de los agentes o individuos ante las señales que recibe. La ontología básica de los PSBE podría entonces caracterizarse como una tríada: señales, agentes y actividades (o reacciones de los agentes).

El aspecto secuencial de un PSBE se asocia a la idea de que los agentes reciben señales del mundo, las interpretan, forman expectativas sobre el valor futuro de alguna (o algunas) variable(s) relevante(s), y llevan a cabo determinada toma de decisiones. Las acciones resultantes provocarán nuevas señales a ser captadas e interpretadas por otros agentes, los cuales, sobre la base de sus expectativas formadas, tomarán determinadas decisiones, y así sucesivamente.

La acción humana juega un rol fundamental en el desarrollo de los PSBE. Específicamente hablando, las personas son agentes activos en al menos dos sentidos. El primero concierne a la recepción de las señales: no se trata de una recepción puramente pasiva o contemplativa, sino que es transformada a partir de un mecanismo de interpretación. En segundo lugar, los actos de las personas, al modificar (o no) ciertos aspectos del mundo, crean nuevas señales, señales que van a ser utilizadas para subsiguientes toma de decisiones. 
Una particularidad de los PSBE es que su estructura básica responde a la lógica de los árboles de posibilidades. Más precisamente, al activarse un determinado factor causal las expectativas formadas - y en consecuencia las acciones llevadas a cabo - pueden ser múltiples. Dependiendo de qué acciones se hayan tomado, distintos serán los resultados sobre el sistema socioeconómico. Así, por ejemplo, sea $X$ la variable independiente (o causa), $Y$ la variable dependiente (o efecto), y $A$ la acción o actividad de las personas. Supongamos que $X$ toma un valor $X_{0}$. Por definición, la variable $Y$ podrá tomar más de un valor $\left(Y_{0}, Y_{1}, \ldots, Y_{\mathrm{n}}\right)$. Todo dependerá de las acciones $A_{1}, A_{2}, \ldots, A_{\mathrm{n}}$ de las personas.

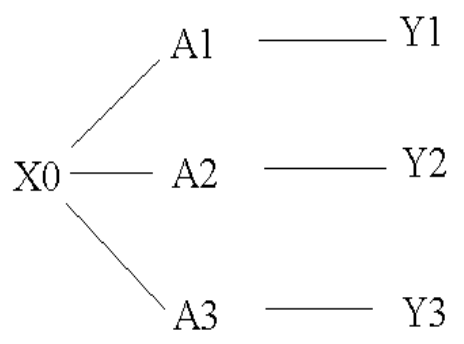

Un claro ejemplo de lo que es un PSBE lo constituye el "efecto Keynes". De acuerdo con este proceso, un aumento en la cantidad de dinero $(M)$ puede conducir a un descenso en la tasa de interés $(i)$, estimulando inversión $(I)$ y en consecuencia el empleo $(N)$ y la producción $(Y)$. La primera etapa (de $M$ hacia i) es generada por un aumento en la oferta monetaria. La explicación tradicional consiste en asumir que las personas aumentarán su demanda de bonos, el precio de este aumentará, y como consecuencia la tasa de interés tenderá a caer. La segunda etapa (de $i$ hacia $I$ ) concierne a la demanda de inversión de las empresas. Si la tasa de interés cae, los créditos serán más baratos para el empresario. Como consecuencia de ello, la inversión aumentará. En lo que respecta a la última etapa (de $I$ hacia $N$ e $Y$ ), Keynes distingue entre ocupación primaria en la industria de inversión $\left(N_{2}\right)$, y ocupación total $(N)$. Cambios en la inversión ocasionan cambios en $N_{2}$. Por otro lado, el multiplicador de Kahn (también denominado multiplicador de la ocupación) muestra cuánto aumentará la ocupación total ante un aumento en $N_{2}$. Supongamos entonces que un aumento en la inversión tiene lugar. Este aumento irá acompañado de un aumento en la ocupación primaria 
en las industrias de inversión. Ceteris paribus, y a través del multiplicador de la ocupación, esto debería aumentar la ocupación total.

El efecto Keynes parece ajustarse bastante bien al esquema de los PSBE, ya que muestra la interrelación entre señales, formación de expectativas y acciones. Una representación simplificada de la estructura subyacente de la primera etapa del efecto Keynes es la siguiente:

$$
\uparrow \mathrm{M} \rightarrow \rightarrow \mathrm{H} \rightarrow \rightarrow \uparrow \mathrm{B}^{\mathrm{d}} \rightarrow \rightarrow \mathrm{i}
$$

donde se pueden identificar con facilidad sus tres componentes básicos: (1) las señales, representadas en este caso por cambios en las variables económicas (aumento en la oferta monetaria $(\uparrow M)$, disminución en la tasa de interés $(\downarrow i))$; (2) los individuos $(\mathrm{H})$, quienes reciben la información (en este caso, del aumento en $M$ ); y (3) las actividades que los individuos desarrollan a partir de la información recibida (aumentan la compra de activos financieros $\left(\uparrow B^{d}\right)$, provocando un cambio negativo en la tasa de interés).

Ahora bien, es erróneo pensar que un cambio en la cantidad de dinero conducirá de manera automática a un cambio inverso en la tasa de interés. Por el contrario, de acuerdo con el marco contextual y con las interpretaciones y expectativas que formen las personas, distintos serán los caminos que puedan tomar esta clase de procesos. Siguiendo con el ejemplo del efecto Keynes, éste no se trata de un proceso aislado, sino que forma parte de un sistema más amplio de factores y procesos conectados entre sí. En consecuencia, el efecto Keynes prevalecerá en la medida en que los cambios que usualmente ocurren en esos otros factores no sean lo suficientemente significativos como para impedir que se cumpla la secuencia de eventos descrita anteriormente. Sin embargo, algunas veces estos cambios sí pueden ser significativos. En tales casos, los individuos modificarán sus cursos de acción, haciendo que efecto Keynes no tenga lugar. Consciente de estas limitaciones, Keynes comenta:

“... si bien puede esperarse que, ceteris paribus, un aumento en la cantidad de dinero reduzca la tasa de interés, esto no sucederá si las preferencias por la liquidez del público aumentan más que la cantidad de dinero; y mientras que puede esperarse que, ceteris paribus, un descenso en la tasa de interés aumente el volumen de la inversión, esto no ocurrirá si la curva de la eficiencia marginal del capital baja con mayor rapidez que la tasa de interés; y mientras es de suponer que, ceteris paribus, un aumento en el volumen de 
la inversión haga subir la ocupación, esto puede no suceder si la propensión marginal a consumir va en descenso." (1936, p.150).

La cita anterior permite mostrar que más que procesos mecánicos, los PSBE son secuencias que pueden comprenderse mejor bajo la lógica de los árboles de posibilidades: dado un acontecimiento determinado, existen diferentes caminos o alternativas. Aquellas alternativas a las cuales se arriben dependerán del cumplimiento de un conjunto de condiciones específicas. Así, en lugar de postular una relación inversa invariante entre oferta monetaria y tasa de interés, debería tenerse en cuenta el hecho de que cambios en la primera pueden conducir a diferentes caminos - por ejemplo, a un aumento en el nivel de precios, a una reducción en la tasa de interés, a ambas, o bien a ninguna de las dos. El resultado al cual se arribe dependerá de las expectativas formadas por las personas respecto del valor futuro de variables económicas relevantes, de las limitaciones - o "habilitaciones" - que impongan ciertas instituciones, etc.

El enfoque de los PSBE es muy semejante al defendido por Cartwright en The Dappled World: A Study of the Boundaries of Science (1999a). En este trabajo Cartwright dirige su crítica al enfoque "legalista" de la ciencia. De acuerdo este último enfoque, el mundo real es un mundo ordenado donde reinan las regularidades. Éste es el legado de aquellos filósofos de la ciencia que toman a la física como modelo a seguir. Cartwright se opone a esta visión de la ciencia. Para la autora, buena parte de lo que ocurre en la naturaleza ocurre por "casualidad", sujeto a ningún tipo de ley. A causa de esta carencia de leyes, el mundo es tanto "desordenado" como "moteado". No hay ninguna garantía que una regularidad que prevalece hoy se mantenga también mañana.

Existe cierta compatibilidad entre la visión de "mundo moteado" (Dappled World) de Cartwright y el enfoque desarrollado en el presente trabajo. En primer lugar, se entiende al mundo real como un sistema donde no hay per se conexiones regulares o conjunciones constantes de eventos. Si estas aparecen, es porque ciertos factores permanecieron invariantes en ese momento del tiempo. Asimismo, el concepto de "mundo moteado" enfatiza la importancia del dominio o rango de aplicabilidad en el cual los modelos son comprendidos. Más precisamente, según esta doctrina es posible que varias teorías y/o modelos - aparentemente contradictorios - sean simultáneamente "verdaderos", siempre y cuando pertenezcan a dominios completamente diferentes (Suarez, 2009). Así, para cada situación tendremos un modelo que proporcionará información acerca de las condiciones se necesitarían cumplir en el mundo real para llegar a un resultado. 
Un modelo sería entonces un "recorte de la realidad”, el cual podrá servir como anteproyecto para cerrar el gran árbol de posibilidades de los sistemas socioeconómicos y llegar así al resultado buscado. Ahora bien, si los modelos representan "cierres" de árboles de posibilidades, entonces - como bien señalaba Cartwright - modelos en apariencia incompatibles pueden no serlo, en tanto y en cuanto sus respectivos rangos de aplicabilidad sean diferentes. Nótese que en estos casos no parece adecuado hablar de veracidad o falsedad en los modelos, sino de aproximación a la realidad de acuerdo con un determinado momento. La existencia de diferentes modelos respecto de una misma temática no implica su incompatibilidad, ya que éstos tan solo representan recortes del mundo real o cierres de un conjunto amplio de alternativas posibles.

\section{Modelando los PSBE. ¿Representaciones aisladas o construcciones ficticias?}

El enfoque de los modelos como aislamientos teoréticos presupone una ontología de factores causales estables como los mecanismos (Mäki, 1992, 2009, 2011) o las capacidades (Cartwright, 1998, 1999a). Puesto que dichos mecanismos (supuestamente) existen en los sistemas socioeconómicos, el científico puede apelar a herramientas puramente teoréticas para obtener la contribución pura del factor bajo análisis.

Sin embargo, es dudoso que este aislamiento sea posible en aquellas situaciones en donde lo que prevalece son los árboles de posibilidades en lugar de los mecanismos. De acuerdo con este enfoque, no hay una secuencia predeterminada que conduzca de una causa $X$ hacia un efecto $Y$. Al activar $X$ podrá ocurrir $Y_{1}$, $Y_{2}, \ldots, Y_{\mathrm{n}}$. Para que cualquiera de estos resultados ocurra deberán cumplirse con una serie de condiciones adicionales. No hay un proceso de "separación" o de "neutralización" de elementos perturbadores, sino de "adición" de nuevos factores condicionantes. ${ }^{4}$ El ejemplo del efecto Keynes mencionado anteriormente es claro: no se trata de un mecanismo por el cual si neutralizamos todos los factores perturbadores pasaremos de un estado inicial de aumento en la cantidad real de dinero a un estado final con aumento en la renta nacional. Para que esta secuencia sea plausible, varias condiciones deberán cumplirse: que la tasa de interés esté en sus valores "normales", que los empresarios tengan buenas expectativas de ventas futuras, que las familias no disminuyan su propensión marginal a

\footnotetext{
${ }^{4}$ Una tesis similar es planteada en Cartwright (2009).
} 
consumir, que haya confianza en la moneda que se está emitiendo, etc. De no darse cualquiera de estas restricciones, la secuencia se desviará por otro camino y el resultado esperado nunca aparecerá.

El enfoque de las construcciones ficticias - y particularmente el de "mundos creíbles" defendido por Sugden $(2002,2009)$ - es diferente. En éste no hay un compromiso ontológico de factores causales estables como los mecanismos o las capacidades. Un mundo creíble puede hacer referencia a un mecanismo que existe - o puede existir - en el mundo real, pero no necesariamente siempre que se construya un mundo paralelo creíble se hará referencia a un mecanismo. El compromiso es más débil. Simplemente se dice que un factor $F$ produce un resultado $R$ en el mundo modelo, y dado que este modelo es creíble, parece razonable pensar que $F$ tenderá a producir $R$ en el mundo real. Sin embargo, F no es un factor aislado - al menos no necesariamente-. ${ }^{5}$ No hay un proceso de "separación" de factores perturbadores. Lo que se hace es construir un escenario posible, que puede ser creíble si es consistente con nuestro conocimiento disponible.

De lo anterior se puede inferir que, en el marco de una construcción ficticia, el modelador puede hacer un recorte de la realidad, cerrando el árbol de posibilidades a través de la adición continua de condiciones antecedentes. En otras palabras, los supuestos que forman parte de un modelo pueden ser entendidos no sólo como un conjunto de premisas que garantizan la inferencia deductiva de resultados, sino también como aquellas condiciones que enmarcan un escenario posible. Suponer que la tasa de interés está en sus valores normales, que la propensión marginal a consumir está constante, etc., no sólo permiten inferir que hay una asociación causal positiva entre cantidad real de dinero y renta nacional; también muestran uno de la miríada de escenarios posibles que se pueden seguir luego de aplicar una política monetaria expansiva.

El conocimiento proporcionado por los modelos no es entonces un mero conocimiento de "teoremas" sin ninguna repercusión en el mundo real (como sí supone Hausman, 1992). Los modelos nos brindan información sobre un conjunto de condiciones que, de satisfacerse en el mundo real, permitiría la aparición de determinados resultados (que son las inferencias del modelo). No es que exista en el mundo real una capacidad social o económica que diga que el aumento en la oferta monetaria conlleva a un incremento en el nivel de renta nacional. Por

5 De hecho, Sugden es explícito en afirmar que gran parte de los modeladores no aíslan factores teoréticamente. 
el contrario, ciertas condiciones tienen que cumplirse para que ello se dé así y no de otra manera. Ahora bien, es importante tener en cuenta el hecho de que dicho cumplimiento no garantiza la aparición de resultados en el mundo real. Por definición, éste es un sistema abierto (véase Lawson, 1997). Esto significa que cualquier elemento exógeno no previsto puede alterar el sendero buscado hacia otros nodos del árbol de posibilidades. Por consiguiente, la asociación causal encontrada en el modelo deberá entenderse en un sentido "tendencial": de cumplirse tales y tales condiciones en el mundo real, esperaremos que emerja un resultado R. Sin embargo, este resultado no estará garantizado.

\section{Referencias bibliográficas}

Alexandrova, A. (2008). "Making Models Count", Philosophy of Science, 75, pp. 383-404.

Boumans, M. (1997). "Lucas and artificial worlds". En J. Davis (Ed.), New Economics and its History. Durham: Duke University Press, pp. 63-88.

Boumans, M. (2006). "The Difference Between Answering a 'Why'-Question and Answering a 'How Much'-Question”. En J. Lenhard, G. Küppers y T. Shinn (Eds.), Simulation: Pragmatic Constructions of Reality. New York: Springer, pp. 107-124.

Bunge, M. (2004). "How Does It Work? The Search for Explanatory Mechanisms". Philosophy of the Social Sciences, Vol. 34, No. 2, pp. 182-210.

Bunge, M. (2005). Buscar la filosofía en las ciencias sociales. Buenos Aires: Siglo XXI editores.

Cartwright, N. (1989). Nature's Capacities and Their Measurement. Oxford: Clarendon Press.

Cartwright, N. (1998). “Capacities”. En J. Davis, W. Hands, y U. Maki (Eds.), The handbook of economic methodology. Cheltenham: Edward Elgar, pp. 45-48.

Cartwright, N. (1999a). The Dappled World. Cambridge: Cambridge University Press.

Cartwright, N. (1999b). "The Vanity of Rigour in Economics: Theoretical Models and Galilean Experiments". Centre for Philosophy of Natural and Social Science. Discussion. También publicado en Cartwright (2007a), Hunting causes and using them: Approaches in philosophy and economics, pp. 217-261. Cambridge: Cambridge University Press. 
Cartwright, N. (2009). "If no capacities then no credible worlds. But can models reveal capacities?”. Erkenntnis, Vol. 70, No.1, pp. 45-58.

de Donato Rodriguez, X. y Zamora Bonilla, J. (2009). "Credibility, idealisation, and model building: An inferential approach”. Erkenntnis, Vol. 70, No. 1, pp. 139-5.

Dow, S. (2002). Economic Methodology: An Inquiry. Oxford: Oxford University Press.

García, R. (2006). Sistemas Complejos. México: Ed. Gedisa.

Gibbard, A. y Varian, H. (1978). "Economic Models", The Journal of Philosophy, Vol. 75, pp. 664-677.

Giere, R. (2004). "How models are used to represent reality". Philosophy of Science, Vol. 71, No. 5, pp. 742-752.

Glennan, S. (2002). "Rethinking Mechanistic Explanation”. Philosophy of Science, Vol. 69, pp. S342-S353.

Glennan, S. (2008). “Mechanisms”. En S. Psillos y M. Curd (eds.), The Routledge Companion to Philosophy of Science. Abingdon: Routledge, pp. 376-384.

Guala, F. (2005). The Methodology of Experimental Economics. Cambridge: Cambridge University Press.

Haavelmo, T. (1944). "The Probablity Approach in Econometrics”. Econometrica, 12 (Supplement), pp. i-viii, 1-118.

Hands, D. (2001). Reflection without Rules. Economic Methodology and Contemporary Science Theory. Cambridge: Cambridge University Press

Hausman, D. (1992). The Inexact and Separate Science of Economics. Cambridge University Press, Cambridge.

Hayek, F. (1981). "La teoría de los fenómenos complejos”. Estudios Públicos, pp. 100127.

Hedström, P. (2005). Dissecting the Social. On the Principles of Analytical Sociology. Cambridge: Cambridge University Press.

Hedström, P. y Swedberg, R. (eds.) (1998a), Social Mechanisms. An Analytical Approach to Social Theory. Cambridge: Cambridge University Press.

Hedström, P. y Swedberg, R. (1998b). "Social mechanisms: an introductory essay”. En P. Hedström y R. Swedberg (eds.), Social Mechanisms: An Analytical Approach to Social Theory. Cambridge: Cambridge University Press, pp. 1-31.

Hedström, P. e Ylikoski, P. (2010). "Causal Mechanisms in the Social Sciences". Annual Review of Sociology, Vol. 36, No. 1, pp. 49-67. 
Hesse, M. (1966). Models and Analogies in Science. Notre Dame: Notre Dame University Press.

Keynes, J. ([1936] 2001). La Teoría General de la Ocupación, el Interés y el Dinero. Buenos Aires: Fondo de cultura económica.

Knuuttila, T. (2005). "Models, Representation, and Mediation”. Philosophy of Science, vol. 72, No. 5, pp. 1260-1271.

Knuuttila, T. (2009). "Isolating representations versus credible constructions? Economic modelling in theory and practice”. Erkenntnis, Vol. 70, No. 1, pp. 59-80.

Lawson, T. (1997). Economics and reality. London: Routledge.

Lawson, T. (2003). Reorienting economics. London: Routledge.

LucAs, R. (1980). "Methods and problems in business cycle theory”. Journal of Money, Credit and Banking, Vol. 12, pp. 696-715.

MäkI, U. (1992). "On the Method of Idealization in Economics”. Poznan Studies in the Philosophy of the Sciences and the Humanities, Vol. 26, pp. 319-354.

MäKI, U. (ed.) (2002a). Fact and Fiction in Economics. Models, Realism, and Social Construction. Cambridge University Press. Nueva York.

MäкI, U. (2002b). “The dismal queen of the social sciences”. En Mäki 2002a (ed.), Fact and Fiction in Economics. Models, Realism, and Social Construction. Cambridge University Press. Nueva York, pp. 3-32.

MÄKI, U. (2005). “Models are Experiments, Experiments are Models”. Journal of Economic Methodology, Vol. 12, No. 2, pp. 303-315.

MäKI, U. (2009). "Missing the world: Models as Isolations and Credible Surrogate Systems". Erkenntnis, Vol. 70, No. 1, pp. 29 - 43.

MäKI, U. (2011). "Models and the locus of their truth". Synthese, Vol. 180, No. 1, pp 47-63.

Marqués, G. (2014). “Tipos de modelos en economía”. Documento de trabajo. $\quad$ http://ciece.com.ar/ciece/wp-content/uploads/Tipos-de-modelos-eneconom $\%$ C3\%ADa.pdf

McMullin, E. (1968). “What Do Physical Models Tell Us?”. En B. van Rootselaar y J. F. Staal (eds.), Logic, Methodology and Philosophy of Science III. Amsterdam: North Holland, pp. 385-396.

Mill, J. ([1843] 1850). A System of Logic. New York: Harper and Brothers. Edición reproducida en R. Backhouse (ed.), The Methodology of Economics: Nineteenth Century British Contributions, Routledge-Thoemmes Press, London, 1997. 
Morgan, M. (2002). "Models, stories, and the economic world". En Uskali Mäki (ed.), Fact and Fiction in Economics: models, realism and social construction. Cambridge: Cambridge University Press, pp. 178-201.

Morgan, M. (2012). The World in the Model. How Economists Work and Think. Cambridge University Press.

Morgan, M. y Morrison, M. (1999). Models as Mediators. Cambridge: Cambridge University Press.

Morgan, M. y Knuuttila, T. (2012). "Models and Modelling in Economics". En U. Mäki (ed.), Handbook of the Philosophy of Economics, Elsevier, Amsterdam, pp. 49-87.

Morrison, M. y Morgan, M. (1999). "Introduction". En Morrison, Margaret y Mary Morgan (eds.), Models as Mediators: Perspectives on Natural and Social Science. Cambridge: Cambridge University Press, pp. 1-9.

Skipper, R. y Milstein, R. (2005). “Thinking about evolutionary mechanisms: natural selection". Studies in History and Philosophy of Biological and Biomedical Sciences, Vol. 36, No. 2, pp. 327-347.

Suárez, M. (2004). "An Inferential Conception of Scientific Representation". Philosophy of science, vol. 71, no. 5, pp. 767-779.

Suarez, M. (2009). "La filosofía de la ciencia y de la economía de Nancy Cartwright". En J. García-Bermejo (ed.), Sobre la Economía y sus Métodos, Enciclopedia Iberoamericana de Filosofía. Editorial Trotta, pp. 383-394.

Sugden, R. (2000). "Credible worlds: the status of theoretical models in economics". Journal of Economic Methodology, Vol. 7, No. 1, pp. 1-31. Reproducido también en U. Mäki (ed.), Fact and Fiction in Economics. Models, Realism, and Social Construction. Cambridge University Press. Nueva York, 2002, pp. 107-136.

Sugden, R. (2009). "Credible worlds, capacities and mechanisms". Erkenntnis, Vol. 70, No. 1, pp. 3-27.

Von Mises, L. (1949). Human Action. A treatise on Economics. Bettina Bien Greaves. http://mises.org/books/humanactionscholars.pdf

Recibido: 7/12/2014

Aceptado: 25/02/2015

Este trabajo se encuentra bajo una licencia de Creative Commons ReconocimientoNoComercial-SinObraDerivada 4.0

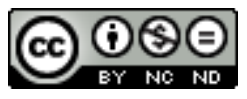

